\title{
Revision amerikanischer Polydesmiden
}

von

\author{
Dr J. GARL
}

Assistent am Naturhistorischen Museum Genf.

Hiezu Tafel 16 und 17.

Gelegentlich der Neuaufstellung unserer Myriapodensammlung sah ich mich veranlasst, die Polydesmiden, welche H. DE SAussure und A. HumberT in ihren vortrefflichen Monographien beschrieben haben, soweit sie noch vorhanden sind, auf den Bau der Copulationsfüsse zu untersuchen. Ein Teil der Ergebnisse ist schon in meiner Arbeit " Exotische Polydesmiden " (Revue Suisse de Zoologie, Tome 10, 1902) enthalten ${ }^{1}$, eine Anzahl Arten werden im Folgenden besprochen, und der Rest wird Gegenstand eines nächsten Aufsatzes werden. Leider ist ein grosser Teil der betreffenden Originalexemplare nicht mehr aufzufinden, sei es dass sie in andre Museen gewandert, oder bei der für diese

${ }^{1}$ Es wurden dort die Beschreibungen der folgenden Arten ergänzt: Icosidesmus Hochstetteri Humb. et Sauss., Pachyurus granosus Humb. et Sauss., Platyrrhacus annectens (Humb. et Sauss.), Platyrrhacus javanus (Sauss.), Platyrrhacus insularis (Humb. et Sauss.), Sphaeriodesmus mexicanus (Sauss.), Cyclodesmus aztecus Humb. et Sauss. 
Tiere durchaus ungeeigneten Methode der trockenen Konservierung zu Grunde gegangen sind. Von andern Arten bleiben nur noch qübrig.

Im Allgemeinen konnte ich mich auf die Beschreibung der Copulationsfüsse beschränken; die übrigen Körperformen hätte ich in den meisten Fällen kaum treffender zeichnen können, als es Humbert und Saussure schon gethan. Diese Autoren trifft auch kein Vorwurf, wenn wir heute eine etwas andere Gruppierung ihrer Formen vorzunehmen genötigt sind, war doch zu ihrer Zeit der hohe Wert der Copulationsfüsse für die Klassifikation noch nicht erkannt.

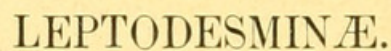

Gattung Eurydesmus Sauss. (nec AtTems 1899).

Eurydesmus angulatus Sauss.

Taf. 16, Fig. 1-6.

H. De Saussure, Mem. Mexique, Myriapodes, p. 78, Tafel 4, Fig. 23, 1860. Humbert et Saussure, Mission scient. au Mexique, p. כ̌.̆, 1872.

Atтems, System der Polydesmiden II., p. 14 [261 1899.

Brölemaxx, H.-W., Myriapodes du Musée de Saó Paulo. Revista do Museo Paulista. Vol. V, p. 93, Pl. V, Fig. 93-96, 1902.

Ein $\sigma^{\top}$ unserer Sammlung erweist sich bei Vergleich mit dem weiblichen Originalexemplar als hiehergehörig. Er stimmt äusserlich mit dem Weibchen in den wesentlichsten Punkten überein.

Prozoniten, Vorderrand der Metazoniten und Kiele gelblich; der grösste Teil der Metazoniten, der Kopf und der ganze Halsschild rotbraun bis weinrot. Unterseite heller braun. 
Antennen bis zum Hinterrand des zweiten Metazoniten reichend, am Ende leicht keulig verdickt. Form der Kiele und Skulptur des Rückens wie beim $\supsetneq$. Ventralplatten (ATTEMs loc. cit.) wie beim $Q$, mit je einem kleinen, spitzen Kegel innen neben der Insertion jedes Beines und einem bogenförmigen Eindruck in der hinteren Hälfte. Ventralplatte des 5. Segments in der Mitte zu einem am Ende in 4 Knöpfchen abgeteilten Höcker erhoben, diejenige des 6. Segmentes am Vorderrande zu einer Querleiste erhöht. Diejenige des 15. Segmentes ohne Fortsatzbildungen (vgl. die $\sigma^{\Upsilon}$ der Euryzonus-Arten).

Beine mit dickem, fein quergestreiftem Haftpolster an der Basis des letzten Gliedes (Fig. 4 und 5).

Copulationsfüsse kurz und stark (Fig. 1-3, 6). Hüfte dick, cylindrisch, auf der Aussenseite den Schenkel mit einem breiten runden Lappen teilweise bedeckend. Schenkel trapezförmig, überall gleichmässig stark und lang beborstet, innen in der Umgebung der kleinen rundlichen Samengrube zu einer runden Beule erhöht. Der Schenkel trägt einen starken Schenkelfortsatz (Sf), der die Länge des Tibialteils erreicht und diesen von aussen fast vollkommen bedeckt; er beginnt mit einem kurżen, dreikantigen Basalabschnitt, auf den ein löffelförmiger, die Concavität nach innen kehrenden Teil folgt. Vom Basalteil des Schenkelfortsatzes entspringt unterseits ein fein gabelig verzweigter, cylindrischer Chitinfortsatz und etwas weiter distalwärts ein flacher, dreieckiger Fortsatz. Die äussere, convexe Fläche des Löffels trägt einen kurzen niederliegenden Dorn, die innere, concave Fläche kurze Reihen feiner Zähnchen. Der gegen den Schenkel deutlicher abgegrenzte Tibialteil (Fig. 6) ist sichelförmig gebogen. Etwa in der Mitte giebt er den kurzen cylindrischen Hauptast ab, der die Samenrinne führt und am Ende in einige sehr fein verzweigte und gezähnelte Aestchen ausgeht. Von der Abgangsstelle des Hauptastes an wird der Tibialteil kahnförmig; die Ränder des Kahnes sind fein gezähnelt und zer- 
schlitzt; der innere trägt einen breiten, blattförmigen, zerschlitzten Fortsatz, der den Hauptast von innen fast gänzlich verdeckt, der äussere einen ganzrandigen, lamellären Fortsatz. Für die Details in den Umrissen muss auf die Abbildungen verwiesen werden.

Für beide Geschlechter gilt noch folgendes: Pleuralkiel an allen Metazoniten gut entwickelt als eine schräg nach hinten und oben ziehende Leiste. Die die Quernat nach vorn begrenzende Leiste ist auf der Unterseite der Segmente viel stärker und schärfer als auf der Oberseite; vor der Ansatzstelle der Beine biegt sie jederseits stark nach vorn, dahinter auf der eigentlichen Ventralfläche ist sie regelmässig nach hinten gebogen.

Anmerkung: AtTEms hat, bevor das ơ von Eurydesmus angulatus bekannt war (Syst. d. Polydesmiden II. pag. 13 [263]), die Gattung Eurydesmus in zwei Untergattungen gespalten und dem Subgenus Eurydesmus die amerikanische Art, dem Subgenus Euryzonus die afrikanischen Formen zugewiesen. Gestützt auf das $\sigma^{\top}$ von E. angulatus ist man genötigt, diese zwei Untergattungen zu zwei sehr distinkten Gattungen zu erheben, indem die Copulationsfüsse der amerikanischen Art nach einem ganz andern Typus gebaut sind als diejenigen der afrikanischen Arten. Dort sind sie kurz und gedrungen und wie bei der Mehrzahl der Leptodesmusarten mit einem starken Schenkelfortsatz versehen; der Schenkel ist gegen die Tibia deutlich abgegrenzt. Bei Euryzonus hingegen sind sie lang und umgeschlagen, dabei einfach, ohne Andeutung eines Schenkelfortsatzes; Schenkel und Tibia sind zu einem Stück verwachsen.

Der fleischige Polster, der sich bei den $\sigma^{x}$ von Euryzonus knapp unter der Klaue und beim $\sigma^{\Upsilon}$ von Eurydesmus unter der Basis des letzten Gliedes vorfindet, haben nichts als die Funktion miteinander gemein. Die amerikanische Form erinnert vielmehr auch durch ihren Besitz an gewisse Leptodesmusarten (L. carneus Sauss., contortus [Broel.]). 
Die Verwandtschaftsfrage $e^{1}$ dürtte für Eurydesmus durch den Bau der Copulationsfüsse allein schon entschieden sein. Wir haben in Eurydesmus Sauss. eine typische, durch ihre abweichende Porenformel besonders charakterisierte Leptodesmidengattung.

$1 \sigma^{x}$, Brasilien, Genfer Museum; 1 , Originalexemplar von Saussure, Brasilien.

\section{Gattung Leptodesmus Sauss. \\ Leptodesmus carneus (Sauss.).}

Taf. 16, Fig. 7-9, 12.

Polydesmus carneus Sauss., Linn. entomol. Bd. XIII, p. 324, 18509.

") (Leptodesmus) carneus Sauss., Myriapodes du Mexique, p. 46, Taf. III, Fig. 15̈, 1860.

" (Oxyurus) carneus Sauss., Humbert et Saussure, Miss. scient. au Mexique, p. 41, 1872.

Leptodesmus carneus Sauss., Atтens, System der Polydesmiden I, p. 157 [377], 1898.

Das $\sigma^{\mathbb{1}}$ war bisher unvollkommen beschrieben.

Antennen mässig lang und ziemlich dünn, zurückgelegt bis zum Hinterrand des zweiten Metazoniten reichend.

Ventralplatte des dritten Segments mit zwei niedrigen, aneinandergedrückten Höckerchen, diejenige des vierten Segments

${ }^{1}$ Zeichnungen und Text dieser Beschreibung waren schon hergestellt, als mir Brölemanns Arbeit: Myriapodes du Musée de Sã̃ Paulo (Revista do Museu Paulista, Vol. V. 1902) zukam, worin die Copulationsfüsse von Eurydesmus ebenfalls beschrieben werden. BRöLEMann hat thatsächlich $E$. angulatus vor sich gehabt und auch die Stellung der Gattung richtig aufgefasst. Aus dem Vergleich unsrer Figuren ergeben sich einige Unterschiede, die wohl auf Rechnung der Variation zu setzen sind. Hingegen liegt bezüglich der Ausmündung der Samenrinne bei BröLEMANN ein Irrtum vor. Ich gebe daher hier die ganze Beschreibung in ihrer ursprünglichen Fassung, ohne natürlich damit BröLemanss Priorität irgendwie bestreiten zu wollen.

Rev. Suisse de Zool. T. 11. 1903. 
zwischen dem vorderen Beinpaar mit zwei längeren, der ganzen Länge nach sich berührenden, höckerförmigen Fortsätzen.

Beine von vorn nach hinten bedeutend an Länge zunehmend, die letzten recht lang und schlank. Das zweite Glied trägt vom zweiten bis vorletzten Beinpaar unterseits am Ende einen scharf begrenzten, nackten Knopf (Fig. 12), das vorletzte Glied einen dicken, weit unter das letzte Glied vorragenden Haftpolster (Fig. 9), der gegen das Körperende hin immer schwächer und kürzer wird und an den letzten Beinpaaren fast gänzlich fehlt. Die Beine der ersten Paare sind ringsum ziemlich dicht beborstet; unterseits sind die Borsten länger und bilden in der distalen Hälfte der drei letzten Glieder einen lichten Büschel; caudalwärts wird die Beborstung immer schwächer, an den letzten Beinpaaren sind die drei proximalen Glieder fast nackt, die übrigen spärlich und ganz kurz beborstet.

Pleuralkiel nur auf den 7 ersten Segmenten vorhanden, in Form einer ventralwärts gebogenen Leiste.

Copulationsfüsse (Fig. 7, 8), lang und stark, in situ betrachtet, parallel nach vorne gerichtet, die convexe Fläche des Endteils nach unten kehrend. Hüften dick, cylindrisch, innen am Grunde miteinander verwachsen, gegen das Ende hin oberund unterseits mit einigen längeren Borsten, der Endrand aussen nahe der Oberseite in einen ziemlich langen, dornartigen Fortsatz ausgezogen. Hüfthörnchen lang, stark gebogen. Śchenkel gleichmässig beborstet, die Samengrube von einer Chitinfalte bedeckt. Die Tibia ist sichelförmig gekrümmt, bis zum Ende gleich breit, innen unsymetrisch kahnartig ausgehöhlt. Sie nimmt den ebenfalls sichelförmigen Schenkelfortsatz in die Concavität auf, ihn von unten ganz, von aussen teilweise verdeckend. Nahe der Basis findet sich innen ein breiter, dreieckiger Fortsatz, dessen Spitze etwas nach aussen gekrümmt ist und in eine von zwei starken Wülsten begleitete Rinne des Schenkelfortsatzes eingreift. Gegen das Ende hin trägt die Tibia innen noch 
einen kleinen dornartigen Fortsatz und eine gezähnelte Leiste. Die Samenrinne folgt ihrem convexen Rande und mündet auf der Innenseite kurz vor dem Ende; ein Hauptast ist nicht deutlich abgetrennt. Der Schenkelfortsatz (Fig. 7) ist ebenfalls sichelförmig, gegen das Ende hin verjüngt und in zwei ungleich lange Gabeläste gespalten. Der Aussenfläche sitzen in einer Längsreihe vier älınliche, cylindrische Aeste auf, ein fünfter, basaler, bleibt kurz und höckerförmig. Im basalen Teil trägt der Schenkelfortsatz aussen zwei scharfe, gebogene Wülste, innen eine gezähnelte Zacke.

$2 \sigma^{x}$, Brasilien, Originalexemplare von Saussure.

\section{Leptodesmus placidus Wood.}

Taf. 17, Fig. 18.

Polydesmus (Leptodesmus) placidus Wood., Proc. Acad. Nat, Sc. Philadelphia, p. 9, 1864 .

" Transact. of the American Philos.

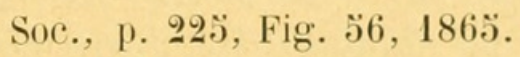
Syn. ” $"$ floridus ” Proc. Acad. Nat. Sc. Philadelphia, p. 9, 1864.

()

") Transact. of the American Philos. Soc. p. 226, 186 .

$\bigcirc^{\Upsilon}$. Länge : $27^{\mathrm{mm}}$. Breite eines Metazoniten der Körpermitte $5^{\mathrm{mm}}$.

Kopf glatt, mit schwacher Scheitelfurche. Antennen zurückgelegt bis zum Hinterrand des zweiten Segmentes reichend.

Halsschild so breit wie der folgende Rückenschild ; seine Seitenlappen dreieckig mit stumpfer Spitze, geradem Vorder- und etwas convexem Hinterrand.

Kiele schmal, im Sinne des Rückens abfallend, mit abgerundetem Vorder- und Hintereck. Vorder- und Hinterrand schmal erhaben gerandet; Seitenrand an den porenlosen Kielen gleich- 
mässig wulstig verdickt, an den porentragenden in der hintern Hälfte zu einer schmal-birnförmigen, vom übrigen Kielrand nicht scharf abgesetzten Beule verdickt.

Rücken verhältnismässig stark gewölbt. Metazoniten glatt und glänzend, ohne oder mit sehr seichtem Quereindruck.

Quernat zwischen Pro- und Metazoniten schmal, bei starker Vergrösserung fein längsgestreift erscheinend.

Seiten des Körpers matt, fein lederartig.

Pleuralkiel vom ersten bis achten Segment nachweisbar.

Ventralplatten glatt, mit feiner Längs- und Querfurche, diejenige des 5. Segments in vier niedrige, stumpfe Höcker geteilt, diejenige des 7 . Segments hinter der Oeffnung für die Copulationsfüsse erhöht.

Beine mässig lang, ziemlich stark und dick, besonders die vorderen Paare, unterseits ziemlich dicht beborstet, mit mehreren längeren Borsten am Ende des ersten und zweiten Gliedes. Die Beine der 6 ersten Segmente sind kürzer und stärker gebaut und auch etwas dichter beborstet als die folgenden; ihr zweites Glied ist oberseits höckerig verdickt.

Analschuppe trapezförmig.

Schwänzchen kurz, kegelförmig.

Von den Copulationsfüssen hat schon Wood (loc. cit. 1865) eine für jene Zeit leidliche Abbildung geliefert, die die Art wiederzuerkennen erlaubt. Sie weichen etwas vom gewöhnlichen Leptodesmustypus ab (Fig. 18). Der Schenkel ist von der Tibia nicht deutlich abgesetzt und besitzt keinen freien Schenkelfortsatz. Der Endteil spaltet sich vor der Mitte seiner Länge in einen langen, geisselförmigen Hauptast (h) und einen komplizierten Nebenast (n). Dieser letztere beschreibt in seiner basalen, lamellären Hälfte eine Spiralwindung um seine Längsachse und weist an dieser Stelle eine starke Zähnelung des Aussenrandes auf; dann geht er in einen verdickten, unterseits stark pinselartig beborsteten, geraden Endabschnitt über, der an der Basis 
einen Lappen (l) nach innen abgibt, und mit demselben eine kurze Scheide für den Hauptast bildet. Möglicherweise entspricht der Nebenast einem ursprünglichen Schenkelfortsatz, der an der Basis sekundär mit der Tibia verschmolzen wäre.

$2 \sigma^{x}$, Vereinigte Staaten (Genfer Museum).

Anmerkung: Die zwei Exemplare unseres Museums stammen wahrscheinlich aus WooD's Sammlung und dürften als Typen angesehen werden. Sie waren als Leptod. floridus etiquettiert; doch fällt diese Art, wie die Copulationsfüsse zeigen, mit L. placidus zusammen.

\section{Leptodesmus subterraneus (Sauss.).}

Taf. 16, Fig. 14.

Polydesmus subterraneus Sauss., Linnæa entomol. XIII, p. 323, 1850.

" " Myriapodes du Mexique, p. 44, Fig. 6, 7, 1860.

Leptodesmus subterraneus Sauss., Atтемs, System der Polydesmiden I, p. 171, [391], 1898.

$\sigma^{\Upsilon}$. Ventralplatte des 5. Segments in 4 stumpfe Höcker geteilt. Diejenige des 7. Segments seitlich und hinter den Copulationsfüssen stark erhöht.

Copulationsfüsse: Hüfte kurz, cylindrisch. Schenkel regelmässig beborstet, aus einem rundlichen, basalen und einem flachen, kurz aufsteigenden, gegen den Endabschnitt durch eine schräge Trennungsfurche scharf abgegrenzten, distalen Teil bestehend. Schenkelfortsatz (Sf) gut entwickelt, zunächst gerade aufsteigend, dann auf der Aussenseite buckelig verbreitert und nach innen gebogen, von der Biegungsstelle an bis zum Ende gleich breit, bandartig, sichelförmig gebogen. Endabschnitt einfach, zunächst leicht s-förmig geschwungen, flach; vom ersten Drittel an etwas keulig verdickt, dann unter plötzlicher Ver- 
schmälerung oral- und basalwärts umgeschlagen und in einen langen, spitzzulaufenden Hauptast ausgehend. Vor der Verschmälerung in den Hauptast, an ihrer breitesten Stelle, trägt die Tibia innen einen kurzen, spitzen Dorn (*).

Originalexemplare von Saussure aus den Grotten von Cuba.

\section{Leptodesmus Couloni (Humb. et Sauss.).}

Taf. 16, Fig. 13 .

Polydesmus Couloni Humb. et Sauss., Revue et Mag. de Zoologie, p. 13̈1, 8, 1869 .

(Oxyurus) Couloni Humb. et Sauss., Miss. scient. Mexique, p. 43, Taf. I, Fig. 9, 1872.

Odontopeltis Couloni Humb. et Sauss., Pocock, Journ. Linn. Soc. XXIV, p. ว̈13, 1894.

Leptodesmus Couloni Humb. et Sauss., Attems, System der Polydesmiden I, p. $170[390], 1898$.

Copulationsfüsse denjenigen von $L$. subterraneus sehr ähnlich. Hüfte und Schenkel wie dort. Schenkelfortsatz (Sf) von der Baśis an gebogen, am Ende stark sichelförmig eingekrümmt, die buckelförmige Auftreibung der Aussenseite näher der Basis als bei $L$. subterraneus. Tibia flach, schwach gebogen, unter plötzlicher Verschmälerung in einen langen, geisselförmigen Hauptast ausgehend, der an seiner Basis eine enge Spirale beschreibt. Ein Dorn vor der Verschmälerung der Tibia an der Innenseite, wie er bei $L$. subterraneus vorkommt, fehlt hier.

Originalexemplar von Saussure. Cuba.

Wie in den Copulationsfüssen, so ähneln sich L. Couloni und L. subterraneus auch in den übrigen Formen stark. Sie unterscheiden sich hauptsächlich durch die Körpergrösse, die breiteren Kiele der $\sigma^{\top}$ bei $L$. Couloni, die schärfer abgegrenzte $\mathrm{Po-}_{0}$ renbeule der $q$ von $L$. subterraneus. 


\section{RHACHIDESMIN \&.}

Unter diesem Gruppennamen fasse ich eine Anzahl Gattungen zusammen, deren Copulationsfüsse des Hüfthörnchens entbehren und auch im Bau der Samengrube besondere Verhältnisse aufweisen. Dieses Kriterium scheint mir ebenso wichtig als der Besitz des Schenkelfortsatzes, auf den die Gruppe der Leptodesminæ gegründet ist. Das Fehlen des Hüfthörnchens ist wohl als sekundärer Zustand anzusehen. Jedenfalls haben wir es hier mit einem Verwandtschaftsmerkmal und nicht mit einer blossen Konvergenzerscheinung zu tun; dafür spricht die sehr beschränkte geographische Verbreitung der Gruppe und der Umstand, dass sich die Copulationsfüsse der hiehergehörigen Formen auch in den übrigen Merkmalen nicht allzusehr von einander entfernen. In der Form und Ausbildung der Kiele herrscht freilich grosse Mannigfaltigkeit. Ebenso variiert die Porenformel. Diese Merkmale zeigen aber bekanntlich oft in ganz unabhängigen Gattungsreihen parallele Entwicklungstendenz und können daher erst innerhalb der Gruppen zur Abtrennung von Gattungen und Untergattungen Verwendung finden. Ein anderes systematisches Merkmal zweiter Ordnung ist die besondere Ausbildung der Samengrube, die jedenfalls erst nach Verlust des Hüfthörnchens stattgefunden hat. Ich fasse die Rhachidesminæ als eine mit den Leptodesminæ nah verwandte Gruppe auf, die, wie Microrhachis uncinatus (Sauss.) anzudeuten scheint, sich von schon mit Schenkelfortsatz versehenen Stammformen abgezweigt hätte. Von da an hätten die beiden Gruppen eine parallele Entwicklung durchgemacht im Sinne der Vereinfachung der Copulationsfüsse durch Reduktion und endlichen Verlust des Schenkelfortsatzes und Verschmelzung von Femur und Tibia. Beim jüngeren Typus der Rhachidesminæ hätten sich diese Modifikationen rascher vollzogen. 
Die hiehergehörigen Gattungen lassen sich unter Beibehaltung der Copulationsfüsse als oberstes Einteilungsprinzip folgenderweise anordnen:

A: Eine deutlich umgrenzte Samengrube fehlt, die ganze Innenfläche der Copulationsfüsse ist zur Aufnahme des Spermas vertieft und lang beborstet Gatt. Aceratophallus Carl.

B. Samengrube vorhanden

a) Samengrube sehr gross, annähernd kreisförmig, ringsum geschlossen, (Copulationsfüsse kurz und gedrungen, Kiele breit, hoch angesetzt)

aa) Poren auf den Segmenten 5, 7-19. Kiele horizontal. Rücken flach. . . Gatt. Strongylodesmus Sauss.

bb) Poren auf den Segmenten 5, 7, 9, 10, 12, 13, 15̃-19, Kiele schräg nach aussen und oben gerichtet, Rücken hohl . . . . . . Gatt. Rhachis Sauss.

1. Kiele breit, lamellär, mit Seitenrand, stark aufgerichtet. . Untergatt. Rhachis s. str. n. subgen.

(Typus Rhachis viridis Sauss.)

2. Kiele dornförmig, mit spitz ausgezogenem Hintereck, Vorder- und Seitenrand zu einem flachen Bogen verschmolzen, Rücken flacher, Habitus Rhachidomorpha-artig

Untergatt. Microrhachis n. subgen.

b) Samengrube rundlich oder unregelmässig, distalwärts weit offen (Copulationsfüsse schlanker, mehrästig, Kiele tiefer angesetzt)

aa) Habitus Leptodesmus-artig, Rücken flach oder schwach gewölbt. Kiele lamellär mit abgerundetem oder sehr stumpfem Hintereck. Halsschild auf den Seiten abgerundet oder zugestumpft

Gatt. Neoleptodesmus n. gen. 
bb) Habitus Strongylosoma-artig, Rücken stark gewölbt, Kiele schmal wulstförmig, ihr Hintereck vom 4 . Kiel an in einen spitzen Dorn ausgezogen. Halsschild a uf den Seiten eckig . . Gatt. Acutangulus Att.

\section{Gattung Strongylodesmus Sauss.}

Strongylodesmus cyaneus Sauss.

Taf. 16, Fig. 10, 11.

Polydesmus cyaneus Sauss., Linnaea entomol. XIII, p. 327, 1859.

Strongylodesmus cyaneus Sauss., Mém. Mexique, Myriapodes, p. 79, Fig. 20, 1860 .

” $"$ Hcmbert et Saussure, Miss. scient. Mexique, p. วั5, 1872.

Aтtems, System der Polydesmiden I, p. 193

[413], 1898.

Copulationsfüsse kurz und gedrungen, einfach. Hüfte kurz, cylindrisch, am Ende nur mit wenigen kurzen Borsten, ohne Hüfthörnchen. Endabschnitt aus einem Stück bestehend, ohne Abgliederung in Schenkel und Tibia. Er stellt einen etwas nach unten gebogenen, dicken Cylinder dar, der innen am Ende sattelförmig eingesenkt ist und in seiner basalen Hälfte eine ziemlich scharf umgrenzte, rundliche, mit längeren Borsten besetzte Vertiefung (Samengrube) aufweist, von der die Samenrinne ausgeht. Der Cylinder setzt sich am Ende in einen dünnen, kurzen, basalwärts umgeschlagenen Ast fort, der die Samenrinne führt. Die ganze Aussenfläche des Cylinders ist gleichmässig beborstet; längere Borsten finden sich längs des Randes der sattelförmigen Einsenkung.

Es stimmt also Strongylodesmus durch den Mangel des Hüfthörnchens, die Form der Samengrube und die gedrungene Gestalt der Copulationsfüsse gut mit Rhachis s. str. überein. 
Auch der äussere Habitus erinnert einigermassen an diese Gattung, doch erlaubt die Porenformel schon eine sichere Trennung. $\sigma^{\top} \sigma^{1}$, Originalexemplare von Saussure, Orizaba Mexico.

Gattung Rhachis Sauss.

Untergattung Microrhachis n. subgen.

Microrhachis uncinata (Humb. et Sauss.)

Taf. 16, Fig. 13.

Polydesmus (Rhachidomorpha) uncinatus Humb. et Sauss., Rev. et mag. de Zool., p. 152, 1869.

Rhachidomorpha uncinata Humb, et Sauss., Miss. scient. au Mexique, p. 38, Fig. 14, 1872.

Die Nachuntersuchung der Originalexemplare dieser Art ergab, dass sie trotz ihres Rhachidomorpha-Habitus doch in Rhachis ihren nächsten Verwandten hat. Diese Auffassung stützt sich auf den Bau der Copulationsfüsse. Um dem grossen Unterschied in der Form der Kiele Rechnung zu tragen (vergl. Bestimmungstabelle), empfiehlt es sich, die Gattung Rhachis in zwei Untergattungen zu trennen: Rhachis s. str. mit Rh. viridis Sauss. als Typus und Microrhachis mit M. uncinata (Humb. et Sauss).

Copula tionsfüsse: Hüfte schwach entwickelt, ohne Hüfthörnchen, Schenkel kurz und breit, innen mit grosser, rundlicher Samengrube, in die distalwärts ein Chitinhöcker einspringt. Ueber die Beborstung lässt sich angesichts des schlechten Erhaltungszustandes des einzigen $\Im^{\Upsilon}$ nichts Sicheres aussagen. Tibia vom Femur nicht deutlich abgegrenzt, geisselförmig, mit birnförmig erweiterter Basis. Oberhalb der Tibia erhebt sich ein stark entwickelter Nebenast (Schenkelfortsatz?), der gegen den Femur ebenfalls nicht deutlich abgesetzt ist und an den Schenkelfortsatz der Leptodesminæ erinnert. Er besteht 
aus 2 Lamellen, wovon die innere einfach, messerklingenförmig, die äussere am Ende verbreitert und mehrfach gelappt ist.

Durch die tiefe Spaltung der Copulationsfüsse bis auf den Schenkel hinab erweist sich $M$. uncinata als Uebergangsform zwischen den Leptodesminæ und Rhachidesminæ. Doch stimmt sie mit letzteren im Bau der Samengrube und im Fehlen des Hüfthörnchens überein.

\section{Gattung Neoleptodesmus n. gen.}

Habitus von Leptodesmus s. str.

Körper aus Kopf und 20 Segmenten bestehend.

Halsschild so breit wie die folgenden Rückenschilder, mit zugerundeten Seitenecken. Kiele flach, horizontal. Vordereck abgerundet. Hintereck bis zum 14. oder 15. Kiele abgerundet, von da an eckig und schwach nach hinten ausgezogen. Poren auf den Segmenten 5, 7, 9, 10, 12, 13, 15-19 auf mehr oder weniger deutlicher Beule.

Rücken fast flach bis ziemlich stark gewölbt. Metazoniten ohne Querfurche, glatt oder längs des Vorderrandes fein gekörnelt.

Ventralplatten breit und kurz. Analschuppe dreieckig mit je einer kleinen Borstenwarze jederseits nahe der Spitze.

Schwänzchen kegelförmig.

Copulationsfüsse lang und stark, von der Mitte an mehrästig. Hüfte ohne Hüfthörnchen. Tibia und Schenkel zu einem Stück verschmolzen, mit breiter, distalwärts offener Samengrube.

Hieher gehören neben «Leptodesmus » Sumichrasti, intermedius Humb. et Sauss. und aztecus Sauss., wahrscheinlich noch mehrere Saussure'sche Formen aus Mittelamerika, beispielsweise L. Orizabe Humb. et Sauss., vielleicht auch L. vermiformis (Sauss.). Doch fehlen mir zur Entscheidung die $\sigma^{\top}$ dieser Formen, deren Copulationsfüsse allein den Ausschlag gäben, da die äusseren Formen mit Leptodesmus übereinstimmen. 
Neoleptodesmus Sumichrasti (Humb. et Sauss.).

Taf. 17, Fig. 22, 23.

Polydesmus Sumichiasti Humb. et Sauss., Revue et Mag. de Zool., p. 151, 1869.

)

Syn. »
(Oxyurus) Sumichrasti Humb. et Sauss. Miss. scient. au Mexique, p. 49, Taf. I, Fig. 8 .

intermedius Humb. et Sauss., Revue et Mag. de Zool., p. 151, 1869.

Miss. scient. au Mexique, p. 49, Taf. I, Fig. 10.

Copulationsfüsse lang und schlank. Hüfte cylindrisch, ohne Hüfthörnchen. Schenkel mit der Tibia vollständig verschmolzen. Samengrube sehr gross, basalwärts und unterseits dick wulstig begrenzt, distalwärts weit offen. Der Boden der Grube ist dicht und lang beborstet. Der Endabschnitt stellt zunächst einen flachen, geraden Cylinder dar; er teilt sich dann ungefähr in der Mitte der ganzen Länge in drei ungleich lange, parallele Aeste. Der mediane Ast, auf den die Samenrinne übergeht, ist der längste und stellt eine schmale, leicht sichelförmig gebogene, bis am Ende gleich breite, abgestutzte Lamelle dar. Seine Basis ist jederseits von einem bedeutend kürzeren, lanzettlichen Nebenaste $\left(\mathrm{N}\right.$ und $\left.\mathrm{N}^{1}\right)$ eingefasst. Der innere Nebenast ist etwas länger als der äussere, sein oberer Rand trägt einige stärkere Kerbzähne; der äussere Nebenast ist etwas kürzer, ganzrandig, längs des Unterrandes ziemlich dicht beborstet.

Die etwas kleineren Exemplare mit schmäleren Kielen, auf welche Humbert und Saussure die Art intermedius gründeten, stimmen im Bau der Copulationsfüsse vollkommen mit Sumichrasti überein und stellen nur eine Variation dieser Form dar.

Originalexemplare von Humbert und Saussure, Orizaba, Mexico. 


\section{Neoleptodesmus aztecus (Sauss.).}

Taf. 17, Fig. 21.

Polydesmus aztecus Sauss., Linn. entom., XIII, p. 324.

Mem. sur le Mexique, Myriapodes, p. 43, Fig. כ..

Die Copulationsfüsse sind denjenigen von N. Sumichrasti sehr ähnlich und nach demselben Typus gebaut, doch stärker und gedrungener. Die basale Hälfte des Endteils ist ziemlich stark nach oben (oralwärts) gekrümmt. Der Hauptast ist kürzer als bei Sumichrasti, am Ende oberseits kaputzenförmig vorgewölbt, abgerundet. Die beiden lateralen Aeste (Nebenäste), besonders der äussere (in der Figur punktierte) sind ebenfalls kürzer und breiter als bei der vorhergehenden Art und erst gegen das Ende hin ziemlich plötzlich zugespitzt; die Zähne am Oberrand des inneren sind weniger zahlreich und dafür stärker.

Für die übrigen nicht unbeträchtlichen Unterschiede zwischen N. Sumichrasti und N. aztecus kann auf die Beschreibungen und Abbildungen der Autoren verwiesen werden. Sie betreffen hauptsächlich die Grösse, die Wölbung des Rückens, Form und Ausbildungsgrad der Kiele und der Porenbeule.

Originalexemplare von Saussure und Humbert. Puebla, Mexico.

\section{Gattung Acutangulus Att.}

ATTEMs hatte schon mit Rücksicht auf den ganzen Habitus, die cylindrische Körperform und die Form der Kiele die hiehergehörigen Formen von Leptodesmus getrennt und eine eigene Gattung dafür geschaffen. Sein Vorgehen erscheint um so berechtigter, wenn man den Bau der Copulationsfüsse in Betracht zieht. Diese ermangeln des Hüfthörnchens und besitzen dafür eine grosse, distalwärts offene Samengrube wie die Gattung Neoleptodesmus; Femur und Tibia sind zu einem Stück verschmolzen, das sich am Ende erst in mehrere kürzere oder längere Aeste teilt: Von Neoleptodesmus unterscheidet sich Acut- 
angulus ebenfalls durch die erstgenannten Merkmale, während die Copulationsfüsse in den beiden Gattungen ähnlich gebaut sind. ATTEMs' Scharfblick hatte die Verwandtschaft schon erkannt, bevor die Copulationsfüsse bekannt waren.

\section{Acutangulus coccineus (Humb. et Sauss.).}

Taf. 17, Fig. 17.

Polydesmus (Tropisoma) coccineus Humb. et Sauss., Revue et Mag. de Zool., p. 152,1869 .

" (Strong!losoma)coccineus »_ " Miss. scient. Mex., p. Ј0, Taf. I, Fig. 12, 1872. Aculangulus coccineus (Humb. et Sauss.) Atтens, System des Polyd. I, p. 189 [409], 1898.

$\sigma^{\Upsilon}$ Die Ventralplatten des 5. und 6. Segments unmittelbar neben jedem Bein in einen zapfenförmigen Höcker ausgezogen.

Copulationsfüsse (Fig. 17) schlank; Endteil leicht gebogen, an der Basis etwas verdickt, am Ende dreiästig. Hauptast schlank und spitz, fast gerade. Nebenäste etwas länger als der Hauptast, leicht sichelförmig basalwärts gekrümmt. Samengrube annähernd kreisförmig, wie der ganze dem Schenkel entsprechende Abschnitt relativ schwach beborstet ${ }^{1}$.

Originalexemplare von Humbert und Saussure, Orizaba, Mexico.

Acutangulus neglectus $\mathrm{n}$. sp.

Taf. 16, Fig. 16 .

Polydesmus (Stronyglosoma) coccineus var. Humb. et Sauss., Miss. scient. an Mexique, p. ว̈1, 1872.

Humbert und Saussure (loc. cit.) erwähnen unter $P$. coccineus zweier reifer $\sigma^{\top}$ von bedeutend geringerer Körpergrösse (13-14 $4^{\mathrm{mm}}$. Die genauere Untersuchung dieser Exemplare ergibt,

${ }^{1}$ Möglicherweise sind bei diesen alten, trocken conservierten Exemplaren die Borsten teilweise weggefallen. 
dass es sich um eine selbständige, der vorigen nahe verwandte Art handelt. Von den Dimensionen abgesehen, habe ich allerdings in den Körperformen keine wesentlichen, messbaren Unterschiede gegenüber cocrineus nachweisen können. Die Ventralplatten des 5. und 6 . Segments tragen beim $\sigma^{x}$ ebenfalls je vier Zäpfchen. Hingegen sind die Copulationsfüsse anders gebaut (Fig. 16). Der Endabschnitt ist kürzer und gedrungener, gerade. Die Samengrube ist basalwärts unregelmässig begrenzt, distalwärts ganz offen, wodurch die Form gewissermassen zum Typus von Aceratophallus überleitet. Die drei terminalen Aeste sind viel kürzer als bei coccineus. Der kurze, in der Richtung der Tibia gerade Hauptast ist am Ende abgestutzt und sehr fein gefranst. Der vordere Nebenast ist etwa von gleicher Länge wie der Hauptast, hakenförmig und schräg nach unten gerichtet. Der dritte Nebenast ist sehr schwach ausgebildet, zahnförmig.

Originalexemplare von Hunbert und Saussure, Orizaba, Mexico.

Die beiden folgenden Gattungen sind bis heute noch in keine bestimmte Gruppe eingeordnet. Die erstere nähert sich Fontaria und damit den Leptodesminæ, die folgende den Oxydesminæ.

Gattung Stenodesmus Sauss.

Stenodesmus mexicanus Sauss.

Taf. 17, Fig. 20.

Stenodesmus mexicanus Sauss., Linn. entomol. Bd. XIII, p. 327, 1839.

) Mem. Mex., Myriapodes, p. 81, 1860.

» Humbert et Saussure Miss. scient. au Mexique, p. รัฐั, 1872.

Das trockene Originalexemplar war leider so schlecht erhalten, dass ich nur den Endteil der Copulationsfüsse untersuchen konnte (Fig. 20). Dieser erinnert sehr an den Typus der Copulationsfüsse der nordamerikanischen Fontaria-Arten, mit denen 
Stenodesmus auch im Besitz eines Dornes am zweiten Beinglied übereinstimmt. Ob im abgebildeten Fragment auch der Femur inbegriffen ist, kann ich nicht entscheiden. Wäre dies der Fall, so würde die beborstete Einsenkung an der Innenseite offenbar der Samengrube der übrigen Polydesmiden entsprechen, und nach ihrer Form könnte man auf den Mangel eines Hüfthörnchens schliessen. Doch bildet dies nur eine Vermutung, die dringend der Bestätigung bedarf.

Originalexemplar von Saussure, Cordova, Mexico.

$$
\begin{aligned}
& \text { Gattung Euryurus C. Koch. } \\
& \text { Euryurus erythropygus (Brdt.). }
\end{aligned}
$$

Taf. 17, Fig. 19.

Polydesmus erythropygus Brandt., Receuil ect., p. 34, 1834.

» maculatus C. Koch, Die Myriapoden, p. 7, Taf. 3, Fig. 8, 1847.

" carolinensis Sauss., Mémoires sur le Mexique, Myriapodes, p. 37, Fig. 3, 1860.

Für die allgemeine Beschreibung kann auf SAussure (loc. cit.) verwiesen werden.

Copulationsfüsse (Fig. 19) einfach gebaut. Hüften kurz und dick, nahe der Tracheentaschen miteinander verschmolzen. Femur abgerundet, unterseits stumpf vorspringend, gegen die Tibia nicht deutlich abgegrenzt. Letztere flach und gerade, erst gegen das Ende hin ziemlich plötzlich rechtwinklig gekrümmt. Spitze des Copulationsfusses kurz gegabelt, in einen dünnen Hauptast und einen kürzeren, hakenförmigen Nebenast ausgehend.

$1 \Im^{\top}$, Originalexemplar von P. carolinensis Sauss. Vereinigte Staaten. 


\section{TAFEL 16.}

\section{FIGURENERKLÄRUNG.}

Fig. 1. Eurydesmus angulatus Sauss. Schenkelfortsatz des Copulationsfusses, von innen.

) $2 . \quad 1$

) $3 . \quad 1$

) 4.4

)) รั.

)

6.
))

)

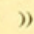

)

》 Copulationsfuss, von innen. ) , von aussen.

Bein des 4. Paares, vom $\sigma^{x}$. Endglieder eines Beines der hintern Körperhälfte, beim $\sigma^{x}$.

Tibia des Copulationsfusses, isoliert, von aussen.

7. Leptodesmus carneus Sauss. Schenkelfortsatz des Copulationsfusses, von aussen und oben.

8. " " Copuiationsfuss, von innen.

9. " " $\quad$ Endteil des 5.. und 6. Glied eines Beines vom $\sigma^{x}$, mit dem Haftpolster.

10. Strongylodesmus cyaneus Sauss. Copulationsfuss, von innen.

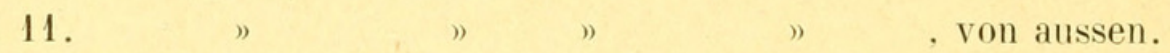

12. Leptodesmus carneus Sáuss. Ende des 2. und Basis des 3. Gliedes des 6 . Beinpaares, vom $\sigma^{x}$.

"13. Leptodesmus C'ouloni (Humb. et Sauss.), Copulationsfüsse, von hinten, aborale Ansicht.

14. ") subterraneus (Sauss.) Copulationsfuss, von hinten.

10ั. Rachis (Microrhachis) uncinata (Humb. und Sauss.) Copulationsfuss, von innen.

" 16. Acutangulus neglectus n. sp. Copulationsfuss, von innen. 


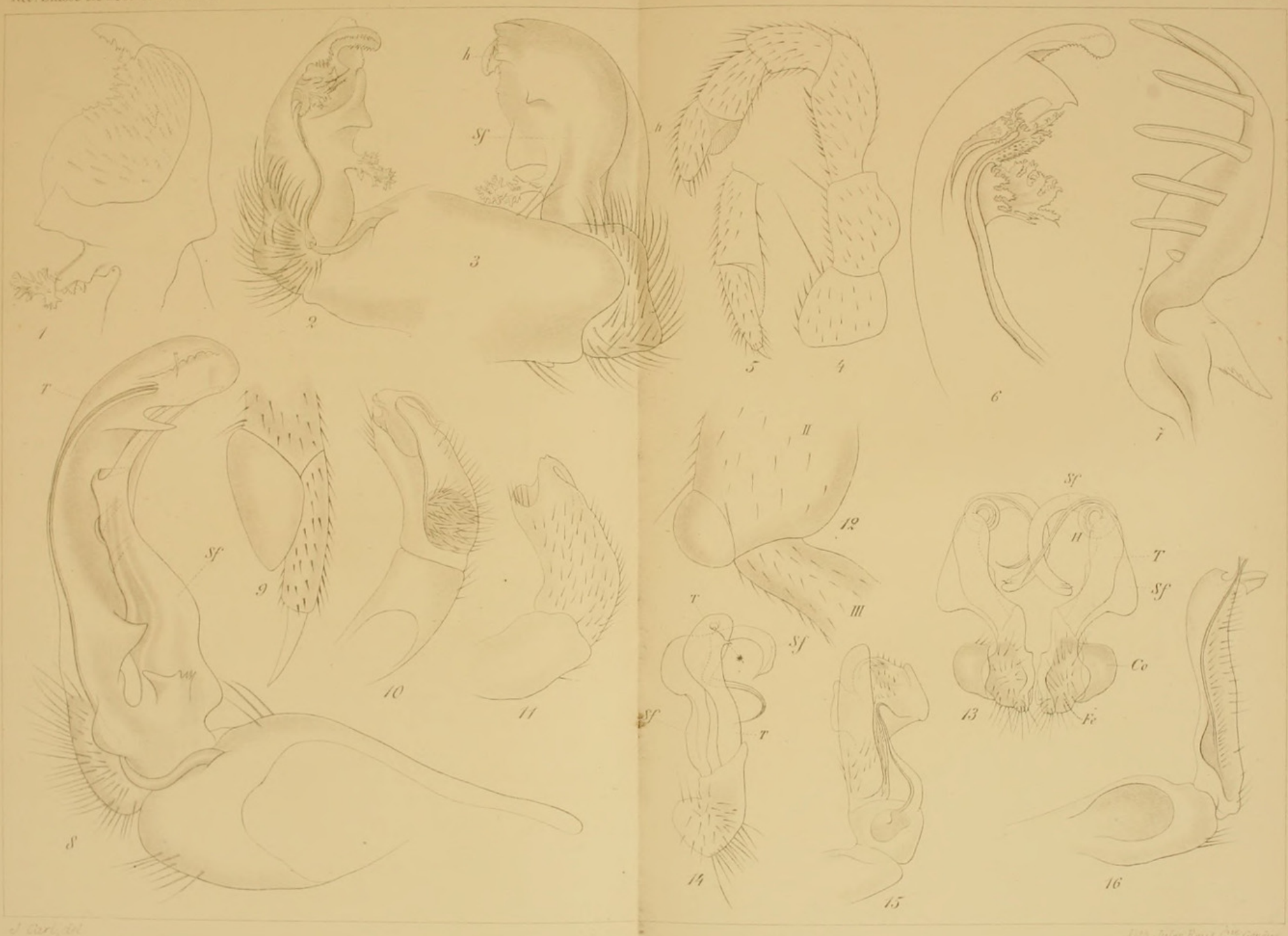

J. Carl._Polydesmiden

F 


\section{TAFEL 17.}

\section{FIGURENERKLÄRUNG.}

Fig. 17. Acutangulus coccineus (Humb. et Sauss.) Copulationsfuss, von innen.

"18. Leptodesmus placidus (Wood). Copulationsfuss, von innen.

) 19. Euryurus erythropygus Brdt.

") 20. Stenodesmus mexicanus Sauss. Endteil des Copulationsfusses, von innen.

"21. Neoleptodesmus aztecus (Sauss.) Copulationsfuss, von innen.

"22. „ Sumichrasti (Humb. et Sauss.) Mittlere Partie des Endteils eines Copulationsfusses mit den Nebenästen, von aussen.

1) 23.

)
(Humb. et Sauss.) Copulationsfuss, von innen. 


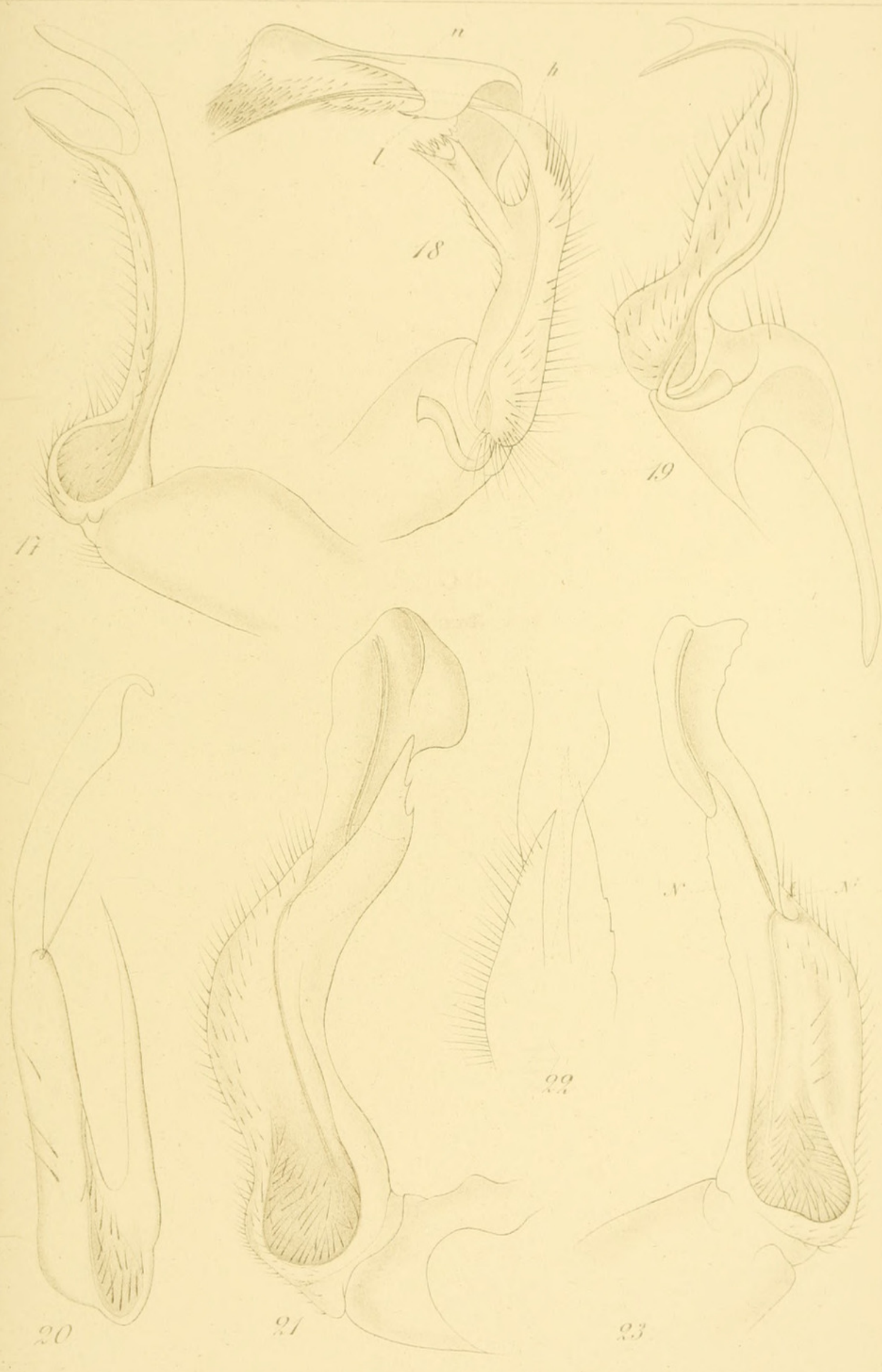

J.Carl.-Polydesmiden. 


\section{$2 \mathrm{BHL}$ Biodiversity Heritage Library}

Carl, Johann. 1903. "Revision amerikanischer Polydesmiden." Revue suisse de zoologie 11, 543-562. https://doi.org/10.5962/bhl.part.82519.

View This Item Online: $\underline{\text { https://www.biodiversitylibrary.org/item/37620 }}$

DOI: https://doi.org/10.5962/bhl.part.82519

Permalink: https://www.biodiversitylibrary.org/partpdf/82519

\section{Holding Institution}

MBLWHOI Library

Sponsored by

MBLWHOI Library

\section{Copyright \& Reuse}

Copyright Status: NOT_IN_COPYRIGHT

This document was created from content at the Biodiversity Heritage Library, the world's largest open access digital library for biodiversity literature and archives. Visit BHL at https://www.biodiversitylibrary.org. 